\section{(A) Check for updates}

Cite this: Food Funct., 2019, 10, 4423

\title{
Effect of Montmorency tart cherry juice on cognitive performance in older adults: a randomized controlled trial
}

\author{
Sheau C. Chai, (D)*a Jessica Jerusik, ${ }^{a}$ Kristina Davis, ${ }^{a}$ Regina S. Wright ${ }^{\mathrm{b}}$ and \\ Zugui Zhang ${ }^{\mathrm{C}}$
}

\begin{abstract}
Hypertension, inflammation and oxidative stress are important factors in the development of cognitive impairment. Our previous study demonstrated that tart cherry juice can lower systolic blood pressure (BP) and improve inflammatory and oxidative stress status in older adults. As part of our previous trial, we explored whether daily consumption of tart cherry juice would improve cognitive abilities. In this randomized controlled trial, 37 adults between the ages of 65-80 with normal cognitive function were recruited and randomly assigned to consume two cups of Montmorency tart cherry juice for 12 weeks. Subjective memory and objective cognitive performance were assessed at baseline and after the 12-week juice supplementation using a validated subjective memory questionnaire and a standardized battery of tests. Daily caloric intake and physical activity levels were assessed throughout the study period. After the intervention, participants in the tart cherry group had higher contentment with memory scores (mean difference of 2.7; $95 \% \mathrm{Cl}: 1.2$ to $4.2 ; p=0.02$ ), lowered their scores of movement time (mean difference of -10.4 ; $95 \% \mathrm{Cl}:-13.4$ to $-7.5 ; p=0.03$ ) as well as performed better on the paired associates learning task (mean difference of $-8.5 ; 95 \% \mathrm{Cl}:-12.5$ to $-4.5 ; p=0.02)$ compared to the control group. The within-group analysis showed that the visual sustained attention $(p<0.0001)$ and spatial working memory $(p=0.06)$ improved after the 12-week consumption of tart cherry juice compared with corresponding baseline values. Daily tart cherry juice consumption may improve cognitive abilities. This may be through anti-oxidative and anti-inflammatory properties of tart cherry and its ability to lower BP. Further research is needed to confirm these findings.
\end{abstract}

Received 29th April 2019, Accepted 9th June 2019

DOI: 10.1039/c9fo00913b

rsc.li/food-function blood pressure (BP) has been strongly associated with increased risk for cardiovascular disease, cerebrovascular disease, cognitive impairment, and dementia. ${ }^{4}$ Multiple studies have shown that elevated BP in individuals in their 40's and 50's raises one's risk of cognitive decline $20-30$ years later. ${ }^{5-7}$ Specifically, for individuals in their $70 \mathrm{~s}$, high BP has been associated with higher risk for MCI. ${ }^{8}$ Findings from the Rancho Bernardo Study showed that for every $5 \mathrm{mmHg}$ increase in systolic BP among older adults, there was a significant decrement in verbal fluency in men and visuomotor tracking and mental flexibility in women. ${ }^{9}$ Although the mechanisms for developing cognitive impairment remain unclear, studies have shown that cognitive impairment in older adults, in part, may be associated with oxidative stress and chronic inflammation. ${ }^{10-12}$

Collective evidence suggests that healthy dietary patterns or consumption of antioxidant-rich foods such as fruit and vegetables is associated with better cognitive performance and reduced risk of cognitive impairment in humans. For instance, meta-analyses and randomized controlled trials have shown that higher adherence to a Mediterranean diet was associated

\footnotetext{
${ }^{a}$ Department of Behavioral Health and Nutrition, College of Health Sciences, University of Delaware, Newark, DE, 19716, USA.E-mail: scchai@udel.edu; Tel: +1 (302) 831-7345

${ }^{b}$ School of Nursing, College of Health Sciences, University of Delaware, Newark, DE, 19716, USA

${ }^{c}$ Value Institute, Christiana Care Health System, Newark, DE, 19718, USA
} 
with lower rates of cognitive decline, reduced risk of cognitive impairment and $\mathrm{AD}$, and improvements in cognitive function. ${ }^{13,14}$ Findings from the Nurses' Health Study showed that higher adherence to the Dietary Approaches to Stop Hypertension diet was associated with better cognitive function in older women. ${ }^{15}$ Numerous studies have found that consumption of fruits rich in anthocyanins and polyphenols, including blueberries, strawberries and grapes, can effectively reduce age-related deterioration in memory and cognition in animals and humans. ${ }^{16-19}$ Tart cherries are a rich source of these flavonoids, especially proanthocyanins, anthocyanins, and flavonols. ${ }^{20}$ Flavonoid consumption has been associated with improvement in cognitive function, in part, due to its ability to reduce $\mathrm{BP}^{21,22}$ and improve cerebral blood flow. ${ }^{23-25}$ In vivo and in vitro studies provide evidence that tart cherries exert anti-oxidative and anti-inflammatory properties. ${ }^{26-30}$ Tart cherries also contain melatonin and potassium..$^{31,32}$ Consumption of tart cherries has been shown to improve sleep performance. Notably, Pigeon et al. ${ }^{33}$ showed that older adults who consumed $480 \mathrm{ml}$ tart cherry juice for two weeks experienced modest, but beneficial effects on sleep. Howatson et $a .^{34}$ demonstrated that total melatonin content and total sleep time were significantly elevated in individuals who consumed tart cherry juice. Another study demonstrated that melatonin exerts anti-amyloidogenic properties which were able to decrease amyloid-beta. ${ }^{35}$ There is emerging evidence that cherries exert neuroprotective effects in animal models. ${ }^{36,37}$ However, little evidence exists for humans. As part of our previously published trial, ${ }^{30,32}$ we explored whether daily consumption of tart cherry juice would improve cognitive abilities in older adults.

\section{Methods}

\subsection{Study design and participants}

The present study was a 12 -week, parallel, randomized controlled trial conducted at the University of Delaware. A detailed description of the study design and participant characteristics have been previously reported. ${ }^{32}$ Briefly, a total of 284 men and women between the ages of 65-80 years were screened for eligibility. During the screening visit, diet information was collected in person via the Block Food Frequency Questionnaire to confirm the inclusion criterion of consuming $\leq 5$ servings of fruits and vegetables per day. Cognitive ability was also assessed using Montreal Cognitive Assessment (MoCA). The MoCA detected MCI with $90 \%$ sensitivity and specificity of $87 \%$ with $95 \%$ confidence interval. ${ }^{38}$ Individuals with a MoCA score $<19$ were excluded from the study. In addition, individuals receiving treatment with any medications that may influence brain function or who had any prior diagnosis or history of stroke, heart disease, diabetes, gastrointestinal disease, cancer, central nervous system or psychiatric disorders, or traumatic brain injury were excluded. Heavy smokers were also excluded from this study. Based on inclusion and exclusion criteria, a total of 37 participants were enrolled $($ men $=17$; women $=20)$ in the study. All participants provided written informed consent before participating in the study. The research protocol was approved by the Human Subjects Committee of the Institutional Review Board at University of Delaware. The trial was registered at clinicaltrials.gov as NCT02922920.

\subsection{Intervention}

A total of 37 men and women (17 in control; 20 in tart cherry) were randomly assigned to consume either two cups (480 ml) tart cherry juice or control drink daily for 12 weeks using a simple randomization and a pre-generated randomization list generated by a computer program. Participants were asked to maintain their habitual diet and physical activity levels during the course of the study. A commercially available Montmorency tart cherry concentrate (King Orchards, MI, USA) was used in the study. The nutrient compositions of the tart cherry concentrate are presented in Table 1 . To prepare $480 \mathrm{ml}$ tart cherry juice, $68 \mathrm{ml}$ Montmorency tart cherry juice concentrate was diluted with $412 \mathrm{ml}$ water. The control drink was prepared by mixing unsweetened black cherry flavored Kool-Aid (Kraft Foods, United States) with water. Dextrose and fructose were added to match the carbohydrate content found in tart cherry. Additional food dye and lemon flavored powder were added to match the color and tartness of the tart cherry juice. Participants were instructed to consume one cup of the beverage in the morning and one cup in the evening for the entirety of the 12-week intervention. Participants were asked to pick up their fresh supplements on a monthly basis. In addition, participants were given customized calendars and were asked to mark the days they missed consuming the study regimen beverage. Unused study regimens were returned for compliance monitoring purposes.

\subsection{Demographics, anthropometric, food record and physical activity}

Demographic information including age, sex, education level, income, race, marital status and employment status was col-

Table 1 Nutrient composition of tart cherry and control beverages

\begin{tabular}{lll}
\hline & $\begin{array}{l}\text { Tart cherry } \\
(480 \mathrm{ml})\end{array}$ & $\begin{array}{l}\text { Control } \\
(480 \mathrm{ml})\end{array}$ \\
\hline Energy (kcal) & 181 & 180 \\
Carbohydrate (g) & 43 & 45 \\
Dietary fiber (g) & - & - \\
Protein (g) & 2.3 & - \\
Fat (g) & - & - \\
Calcium (mg) & 8.6 & - \\
Phosphorous (mg) & 46 & - \\
Potassium (mg) & 355 & - \\
Thiamin (mcg) & 11 & - \\
Folic acid (mcg) & 18.6 & - \\
ORAC (unit) & 6092.8 & - \\
Total tannins (mg) & 95.9 & - \\
Total phenolics (gallic acid equivalents) & 450.6 & -
\end{tabular}

ORAC - oxygen radical absorption capacity. 
lected from participants at the screening visit. Anthropometric, dietary and physical activity assessments were collected at baseline and after the 12-week intervention. Briefly, a stadiometer was used to measure height to the nearest $0.1 \mathrm{~cm}$. Weight was measured in kilograms with minimal clothing on a digital scale to the nearest $\pm 0.1 \mathrm{~kg}$. Body mass index (BMI, $\mathrm{kg} \mathrm{m}^{-2}$ ) was calculated. To monitor dietary intake and physical activity, a 3-day food record (two weekdays and one weekend) and the Physical Activity Scale for the Elderly questionnaire (PASE) were assessed at baseline and after the 12-week dietary intervention. Collected food records were analyzed using Nutrition Data System for Research software (NDSR, Minneapolis, MN).

\subsection{Cognitive tests and subjective memory questionnaire}

Four cognitive domains of the Cambridge Neuropsychological Test Automated Battery (CANTAB, Eclipse Version 3.0) were administered by the trained researcher at baseline and after the 12-week dietary intervention. All tasks were completed in a quiet testing room using a touch screen computer. The paired associates learning (PAL) test assesses episodic visual memory and new learning. In this test, participants were presented with six or eight boxes on the screen, each of which opened up in a random order. The patterns were displayed in the middle of the screen, one at a time. Participants were asked to identify which pattern belonged in which box. This test has an increasing level of difficulty ranging from two to eight patterns to be identified. Rapid visual information processing (RVP) test measures visual sustained attention. A white box appears in the middle of the screen, inside which digits, from 2 to 9, appear in a pseudo-random order at the rate of 100 digits per minute. The participants were instructed to press the response pad when they detect any one of three consecutive odd or even digit sequences (for example, 2-4-6, 3-5-7, 4-6-8). The outcome measures of RVP is RVP $\mathrm{A}^{\prime}$. This is the signal detection measure of sensitivity to the target. Reaction time (RTI) test measures speed of response and movement to a visual target that appears on the screen. Participants were asked to respond to a visual target by using the response pad and/or by touching the screen. Spatial working memory (SWM) test assesses working memory and strategy use. Participants were asked to find a blue token in each of the boxes and then to use them to fill an empty bar on the right-hand side of the screen. Also, digit span (DS) was administered to evaluate short term working memory. Participants were presented with a series of digits and were instructed to immediately repeat them back. Aside from cognitive tests, a self-report memory questionnaire was administered at baseline and after the 12-week dietary intervention to assess subjective memory abilities. This questionnaire assesses three dimensions of self-reported memory including: (1) the overall contentment or satisfaction with one's own memory ability, (2) perception of everyday memory ability, and (3) use of everyday memory strategies. Participants were asked to rate their level of agreement with each subjective item on a 5-point scale according to how they felt over the past two weeks.

\subsection{Statistical analyses}

All analyses were performed using SAS 9.4 (SAS Institute Inc., Cary, NC, USA). Values are reported as mean \pm standard deviation (SD). The student's $t$ test or Wilcoxon rank sum test was used to compare continuous variables at baseline between two groups; paired $t$-test was used to compare the mean changes in outcome variables within the group during the intervention period. Contingency table analysis (chi-square or Fisher exact test) was used to compare categorical variables. To account for the potential effect of baseline factors which were significantly different between two groups on final outcome variables, the 12-week outcomes were estimated via ANCOVA analysis, with adjustment for physical activity, dietary cholesterol, and MoCA which showed significant baseline differences between tart cherry and control groups. The within-group mean changes in outcome variables (12 weeks - baseline) during the intervention period were compared between tart cherry and control using the Wilcoxon rank sum test. Mixed ANOVA model analysis was utilized to examine the impact of group, time, and the interaction of group and time. In all statistical comparisons, differences with $p<0.05$ were considered significant. Sample size calculation was previously reported. ${ }^{30}$ We also reassessed the sample size based on the actual number of participants in this study.

\section{Results}

\subsection{Participants characteristics, food record and physical activity}

A total of 284 individuals were screened over the phone. Following the initial phone screening, 129 potential participants were invited to the study site to complete a screening visit. Based on inclusion and exclusion criteria, a total of 37 participants were enrolled in this study. Thirty-four participants had completed the study. The attrition rate was $8 \%$. Reasons for withdrawal included noncompliance with the study protocol, loss of interest, and an unrelated medical condition. Using G-Power calculation, ${ }^{39}$ a sample size of 34 (17 in each group) would maintain power of $80 \%$ in two-tailed $t$-test with $\alpha=0.05$ and $r=0.50$ between repeated measures for detecting an effect size $d=0.45$ (which is suitable for majority of outcomes) in the group after consumption of tart cherry juice for 12 weeks. Of participants who completed the study, the compliance was $94.2 \%$. No adverse events were reported in the study.

There were no significant differences between the two groups for baseline age $(69.5 \pm 3.9$ years in control; $70.0 \pm 3.7$ years in tart cherry; $p=0.65)$, body weight $(78.7 \pm 13.5 \mathrm{~kg}$ in control; $78.0 \pm 10.3 \mathrm{~kg}$ in tart cherry; $p=0.56)$, height $(169.6 \pm$ $7.9 \mathrm{~cm}$ in control; $165.5 \pm 6.8 \mathrm{~cm}$ in tart cherry; $p=0.25)$, BMI $(27.3 \pm 4.2$ in control; $28.5 \pm 3.7$ in tart cherry; $p=0.34)$, and sex ( 9 males and 8 females in control; 8 males and 12 females in tart cherry; $p=0.43)$ except for MoCA score $(26.7 \pm 2.1$ in control; $24.8 \pm 2.5$ in tart cherry; $p=0.02$ ). In terms of demographics, there were no significant differences between the two 
groups for educational level, income, race, and marital status except for employment status $(p=0.01)$. In the tart cherry group, half of the participants were working while half were retired. In the control group, the majority of participants were retired $(88 \%)$. The majority of the participants had at least a 4 -year college degree (35.5\%), followed by high school $(27 \%)$, advanced degree (24.5\%), and 2-year college degree (13\%). $84 \%$ of participants were White and $73 \%$ of participants were married.

Analysis of 3-day diet records showed that there were no significant differences between the two groups for mean intake of total energy, carbohydrate, protein, fat, and fiber except for dietary cholesterol. The baseline dietary cholesterol was significantly lower in the tart cherry group compared to the control group $(262.0 \pm 133.9 \mathrm{mg}$ in control; $203.0 \pm 90.3 \mathrm{mg}$ in tart cherry; $p<0.05)$. The mean dietary cholesterol significantly increased in both groups $(p<0.05)$. In contrast, from assessment of PASE, the baseline physical activity levels were significantly higher in the tart cherry group compared with the control group $(135.8 \pm 37.3$ in control; $224.5 \pm 190.6$ in tart cherry; $p<0.05)$. The physical activity level significantly decreased in both groups $(p<0.0001)$.

\subsection{Subjective memory and cognitive performance}

The subjective scores of memory ability, contentment with memory, and memory strategy were not statistically different at baseline between tart cherry and control groups (Table 2). After the 12 weeks intervention, participants in the tart cherry group had higher contentment with memory scores than the control group (mean difference of 2.7 ; 95\% CI: 1.2 to 4.2 ; $p=0.02$ ), as shown in Fig. 1 . There were no significant main effects of group, time, or group $\times$ time interaction effects observed for memory ability, contentment with memory and memory strategy scores.

The DS scores were not statistically different at baseline between tart cherry and control groups. The DS scores were significantly different between the two groups after the 12 weeks intervention. The final DS scores in the control group were higher than in the tart cherry group (mean difference of $-0.57 ; 95 \% \mathrm{CI}:-0.81$ to $-0.33 ; p=0.01$ ). In addition, there was a group effect observed for DS $(p=0.0005)$.

The baseline cognitive battery tests of PAL and RTI did not significantly differ between tart cherry and control groups. On the PAL task, there was a significant group effect $(p=0.01)$ and a trend towards a significant interaction of group and time effect observed for PAL $(p=0.09)$. After the 12 -week intervention, participants in the tart cherry group performed better on the PAL task by making fewer errors compared to control group (mean difference of $-8.5 ; 95 \% \mathrm{CI}$ : -12.5 to $-4.5 ; p=$ 0.02), as shown in Fig. 2. On the RTI task, the movement time scores were significantly different between the two groups after the 12-week intervention. Participants in the tart cherry group had lowered their scores of movement time compared to participants in the control group (mean difference of $-10.4 ; 95 \%$ CI: -13.4 to $-7.5 ; p=0.03$ ), as shown in Fig. 3 . There were no group, time, or group $\times$ time interaction effects observed for
Table 2 Subjective memory and cognitive ability scores at baseline and after 12 weeks of tart cherry juice or control drink supplementation

\begin{tabular}{|c|c|c|c|c|c|}
\hline \multirow[b]{2}{*}{ Variables } & \multirow{2}{*}{$\begin{array}{l}\text { Control } \\
(n=17)\end{array}$} & \multirow{2}{*}{$\begin{array}{l}\text { Tart cherry } \\
(n=17)\end{array}$} & \multicolumn{3}{|c|}{${ }^{a} p$-Value (mixed ANOVA) } \\
\hline & & & Group & Time & Intera \\
\hline \multicolumn{6}{|c|}{ Subjective memory } \\
\hline \multicolumn{6}{|c|}{ Memory ability } \\
\hline Baseline & $55.8 \pm 8.6$ & $51.5 \pm 10.3$ & 0.16 & 0.34 & 0.25 \\
\hline 12 weeks & $52.2 \pm 1.8$ & $51.8 \pm 2.6$ & & & \\
\hline \multicolumn{6}{|c|}{ Memory contentment } \\
\hline Baseline & $40.3 \pm 5.0$ & $39.4 \pm 7.0$ & 0.54 & 0.64 & 0.15 \\
\hline 12 weeks & $39.2 \pm 2.7$ & $40.9 \pm 1.2$ & & & \\
\hline \multicolumn{6}{|c|}{ Memory strategy } \\
\hline Baseline & $32.7 \pm 8.2$ & $36.3 \pm 8.0$ & 0.24 & 0.69 & 0.26 \\
\hline 12 weeks & $35.1 \pm 5.3$ & $35.1 \pm 2.5$ & & & \\
\hline \multicolumn{6}{|c|}{ Digit span } \\
\hline Baseline & $29.3 \pm 4.1$ & $26.4 \pm 5.3$ & 0.0005 & 0.60 & 0.68 \\
\hline 12 weeks & $30.1 \pm 1.4$ & $26.5 \pm 2.3$ & & & \\
\hline \multicolumn{6}{|c|}{ PAL first trial memory } \\
\hline Baseline & $19.4 \pm 3.2$ & $17.8 \pm 3.3$ & 0.68 & 0.96 & 0.09 \\
\hline 12 weeks & $18.4 \pm 1.4$ & $18.9 \pm 1.2$ & & & \\
\hline \multicolumn{6}{|c|}{ PAL total errors adjusted } \\
\hline Baseline & $17.3 \pm 11.5$ & $17.1 \pm 10.3$ & 0.01 & 0.64 & 0.09 \\
\hline 12 weeks & $21.9 \pm 12.0$ & $13.2 \pm 3.5$ & & & \\
\hline \multicolumn{6}{|c|}{ RTI movement time } \\
\hline Baseline & $370.4 \pm 54.2$ & $368.6 \pm 40.2$ & 0.23 & 0.52 & 0.32 \\
\hline 12 weeks & $373.6 \pm 27.6$ & $361.4 \pm 15.2$ & & & \\
\hline \multicolumn{6}{|c|}{ RTI reaction time } \\
\hline Baseline & $358.9 \pm 50.4$ & $367.2 \pm 42.7$ & 0.98 & 0.36 & 0.35 \\
\hline 12 weeks & $358.9 \pm 24.7$ & $350.5 \pm 15.0$ & & & \\
\hline \multicolumn{6}{|l|}{ RVP A $\mathbf{A}^{\prime}$} \\
\hline Baseline* & $0.93 \pm 0.05$ & $0.90 \pm 0.03$ & 0.15 & 0.01 & 0.06 \\
\hline 12 weeks & $0.93 \pm 0.02$ & $0.93 \pm 0.01 \dagger$ & & & \\
\hline \multicolumn{6}{|c|}{ RVP mean latency } \\
\hline Baseline & $469.9 \pm 103.7$ & $449.1 \pm 66.1$ & 0.24 & 0.85 & 0.90 \\
\hline 12 weeks & $472.7 \pm 27.6$ & $454.0 \pm 37.2$ & & & \\
\hline \multicolumn{6}{|c|}{ SWM strategy } \\
\hline Baseline $^{*}$ & $30.3 \pm 7.3$ & $35.3 \pm 4.8$ & 0.001 & 0.47 & 0.67 \\
\hline 12 weeks & $31.0 \pm 1.2$ & $34.1 \pm 3.3$ & & & \\
\hline \multicolumn{6}{|c|}{ SWM total error } \\
\hline Baseline $^{*}$ & $20.2 \pm 20.9$ & $44.5 \pm 13.3$ & $<0.0001$ & 0.50 & 0.08 \\
\hline 12 weeks & $23.8 \pm 8.2$ & $36.4 \pm 6.9 \#$ & & & \\
\hline
\end{tabular}

Values are means \pm standard deviation (SD). PAL - paired associate learning; RTI - reaction time; RVP - rapid visual processing; SWM spatial working memory. The estimated 12 -week value was adjusted for baseline physical activity, dietary cholesterol, and MoCA score, assessed by ANCOVA. ${ }^{a} p$-Value for the effects of group, time, and interaction of group and time via mixed ANOVA. ${ }^{*} p<0.05$ for baseline differences between two groups. $₫ p<0.0001$ and $\# p=0.06$ for withingroup differences in comparison with baseline.

RTI movement time and reaction time scores. RTI reaction time scores were not affected by tart cherry or control consumption.

On the RVP task as outcome measure of RVP A' (a measure of how good the subject is at detecting target sequences), as shown in Fig. 4, the within-group analysis revealed that participants in the tart cherry group significantly improved on the RVP task at 12 weeks compared with corresponding baseline values $(p<0.0001)$ whereas there were no changes in the control group. The RVP A' scores were significantly different between the two groups after the 12-week intervention (mean difference of $0.04 ; 95 \%$ CI: 0.01 to $0.07 ; p=0.01$ ). In addition, there was a significant time effect observed $(p=0.01)$ and a 


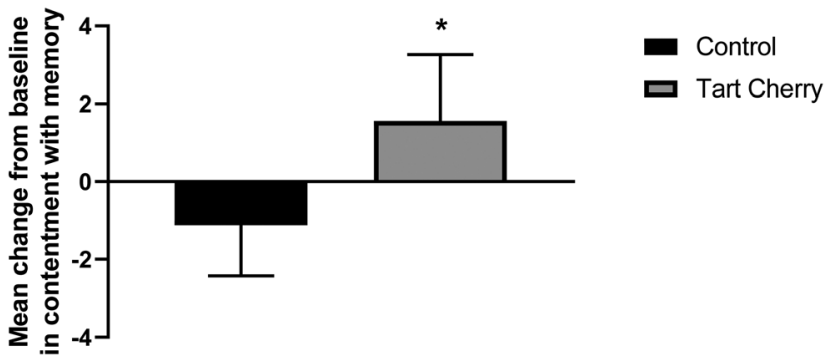

Fig. 1 Change from baseline in contentment with memory (mean \pm standard deviation) by supplementation with control and tart cherry juice. * Mean values were significantly different between two groups ( $p=$ 0.02 ). The difference between two group is the difference of withingroup mean change (12-week value - baseline value).

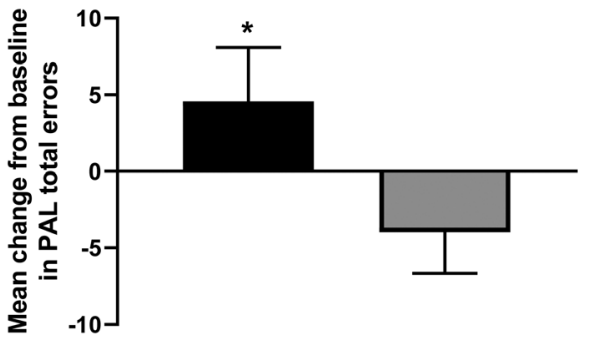

- Control
口 Tart Cherry

Fig. 2 Change from baseline in total errors on paired associates learning (PAL) task (mean \pm standard deviation) by supplementation with control and tart cherry juice. *Mean values were significantly different between two groups $(p=0.02)$. The difference between two group is the difference of within-group mean change (12-week value - baseline value).

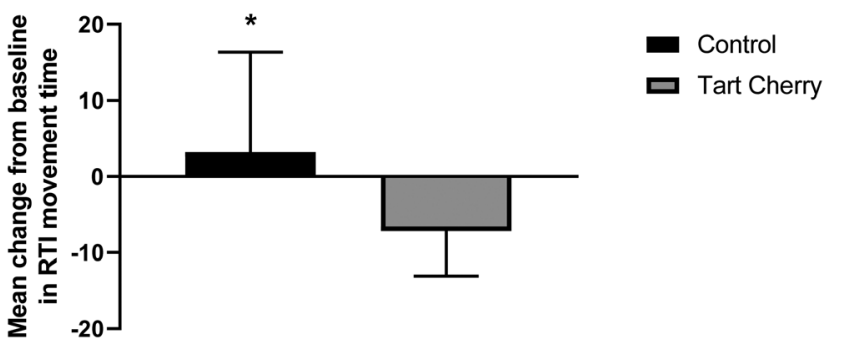

Fig. 3 Change from baseline in movement time (mean \pm standard deviation) by supplementation with control and tart cherry juice. *Mean values were significantly different between two groups $(p=0.03)$. The difference between two group is the difference of within-group mean change (12-week value - baseline value).

trend towards a significant interaction of group and time effect observed for RVP $\mathrm{A}^{\prime}(p=0.06)$. Of note, the baseline scores of RVP $\mathrm{A}^{\prime}$ were different between the two groups $(p<0.05)$.

Similar results were shown for the SWM task, as shown in Fig. 5. The within-group analysis revealed that participants in the tart cherry group showed improvement that approached significance on the SWM task by making fewer errors at 12 weeks compared with corresponding baseline values

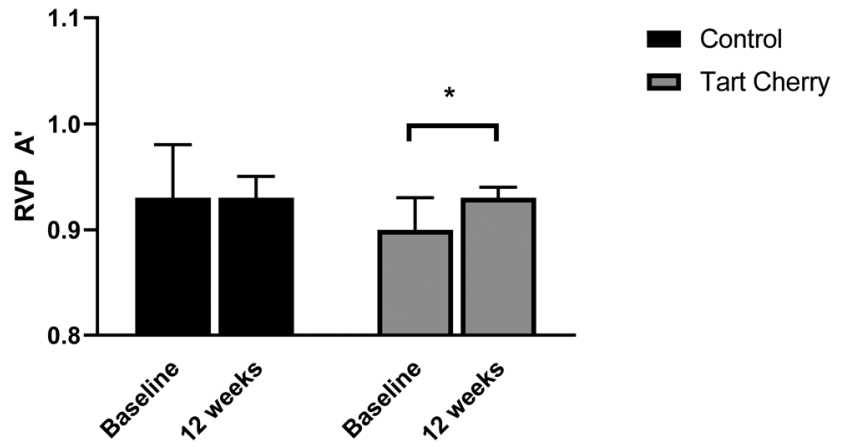

Fig. 4 Rapid visual information processing (RVP) task (mean \pm standard deviation) by supplementation with control and tart cherry juice at baseline and 12 weeks. Participants in the tart cherry group significantly improved visual sustained attention at 12 weeks compared with corresponding baseline values $\left({ }^{*} p<0.0001\right)$ whereas there were no changes in the control group.

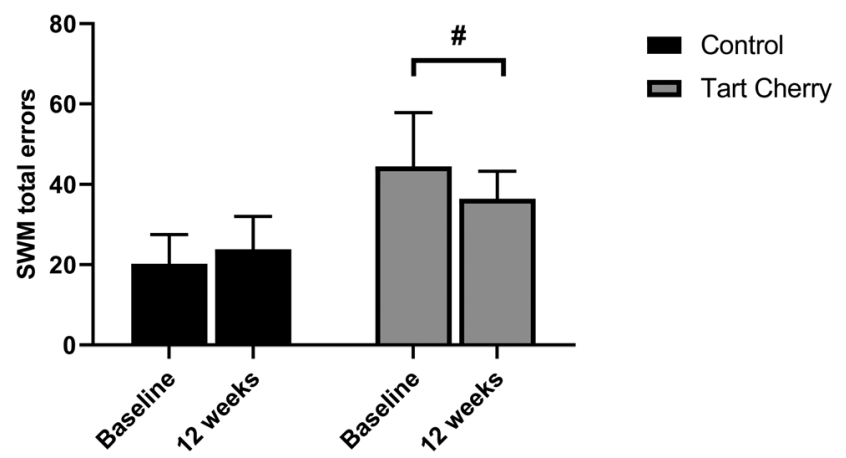

Fig. 5 Spatial working memory (SWM) performance (mean \pm standard deviation) by supplementation with control and tart cherry juice at baseline and 12 weeks. Participants in the tart cherry group significantly improved SWM task at 12 weeks compared with corresponding baseline values (\# $p=0.06)$, whereas there were no changes in the control group.

$(p=0.06)$ whereas there were no changes in the control group. The SWM strategy scores (mean difference of -0.46 ; 95\% CI: -0.57 to $-0.35 ; p=0.02$ ) and SWM total error scores (mean difference of -11.27 ; 95\% CI: -20.82 to $-1.72 ; p=0.03$ ) were significantly different between the two groups after the 12-week intervention. The final SWM strategy and total error scores in the tart cherry group were lower than in the control group. The main effect of group was noted for SWM strategy scores $(p=0.001)$ and SWM total error scores $(p<0.0001)$. In addition, there was a trend towards a significant interaction of group and time effect observed for SWM total error $(p=0.08)$. Of note, the baseline scores of SWM strategy and total errors were different between the two groups $(p<0.05)$.

\section{Discussion}

In our earlier study, ${ }^{32}$ we demonstrated that tart cherry juice can lower systolic BP in older adults. The BP-lowering effect of 
tart cherry juice may be through its anti-oxidative and antiinflammatory properties. $^{30,32}$ This current investigation extends upon our recent study findings to explore the ability of tart cherry juice to improve subjective memory and cognitive function in older adults. Our present study demonstrated that 12 weeks supplementation of tart cherry juice increased subjective memory in the domain of contentment with memory by $5 \%$ and reduced movement time by $4 \%$ in comparison with the control drink. Tart cherry juice supplementation also reduced errors in episodic visual memory by $23 \%$ compared to control drink as assessed by PAL task. Twelve-week consumption of tart cherry juice significantly improved visual sustained attention by $3 \%$ and tended to improve working memory as evidenced by reduction in total errors on SWM task by $18 \%$ at 12 weeks compared with corresponding baseline values. There was no remarkable effect on reaction time, SWM strategy, digit span, and subjective memory ability and strategy.

Aside from our present findings, there is some evidence demonstrating that cherries, both tart and sweet varieties enhance cognition in animal models and humans. Thangthaeng et al. ${ }^{36}$ using a Fischer 344 aged rat model, discovered that rats that were fed a $2 \%$ Montmorency tart cherry diet for six weeks improved working memory. In addition, tart cherry supplementation was also able to reduce neuroinflammatory markers including glial fibrillary acidic protein, NADPH oxidase-2, and cyclooxygenase-2 and improve autophagy function in the hippocampus. Matchynski et al.,${ }^{37}$ using a mu-p75 saporin induced mouse model of $\mathrm{AD}$, found that these mice that were fed a diet containing of 30,60 , or $90 \mathrm{mg}$ $\mathrm{kg}^{-1}$ tart cherry extract and essential fatty acids for four weeks showed decreased cognitive errors related to object recognition, place-recognition, and Morris water maze tasks compared to the control group. In terms of human studies, Kent et al. ${ }^{40}$ demonstrated that 12 -week consumption of $200 \mathrm{ml}$ of sweet cherry juice significantly improved verbal fluency, shortterm, and long-term memory in older adults with mild-to-moderate dementia. However, in both of the acute studies carried out to date, no significant improvement in cognitive performance in humans was detected. ${ }^{41,42}$ Based on our present findings along with others, a minimum of 12 weeks supplementation of cherry juice may be needed to elicit cognitive improvements in older adults because polyphenolic containing compounds may take several weeks to accumulate in brain tissues. $^{43,44}$

The proposed mechanism behind tart cherry's enhancement of cognition may be related to the bioactive compounds present in tart cherries, including various polyphenolic compounds, anthocyanins, and melanin that act as antioxidants. He et al. has previously shown that melatonin exerts anti-amyloidogenic properties which were able to decrease amyloidbeta, a main component of the amyloid plaques found in the brains of $\mathrm{AD}$ patients. ${ }^{35}$ The mechanistic action behind melatonin's antioxidant and neuronal protective properties are thought to be associated with the inhibition of nitric oxide (NO) synthase, stimulating the production of antioxidative enzymes, and reducing free radical production. ${ }^{45}$ Several studies have found that consumption of fruits rich in anthocyanins including blueberries, strawberries, and grapes can effectively reduce age-related deteriorations in memory and cognition in animals and humans. ${ }^{16-19}$ Specifically, Krikorian et al. ${ }^{19}$ has shown that blueberry supplementation improved list recall and paired associates learning scores in participants with mild memory changes. Another human study demonstrated that grape juice consumption for 12 weeks significantly improved verbal learning. ${ }^{46}$ Casadesus et al.,${ }^{47}$ has demonstrated that plasticity is increased in anthocyanin-rich blueberry-fed aged animals. In addition, several anthocyanins were found to be capable of crossing the blood-brain barrier and were localized in neural areas related to memory and processing. ${ }^{44,48}$ Bowtell et al. ${ }^{49}$ found that 12 weeks supplementation of blueberry improved brain perfusion in healthy older adults. Recent studies speculate these improvements are associated with increases in NO synthase. ${ }^{50}$ Interestingly, Lee et al. ${ }^{51}$ and Krikorian et al.,${ }^{18}$ demonstrated that there was significantly increased activation in the right superior parietal cortex in patients with MCI after supplementation with grape. Future studies must examine the mechanisms specific to this part of the brain that are vulnerable to plasticity via tart cherry juice.

Reactive oxygen species (ROS) and superoxide, generated by oxidative stress and low-grade inflammation that accompany aging recapitulate their role in age-associated vascular dysfunction and cognitive impairment. ROS caused by oxidative stress leads to neuroinflammation, and cell death, triggering neurodegeneration and memory decline. ${ }^{52}$ Other proposed mechanism behind anthocyanin-rich foods' attenuation of cognitive decline is by modulation of ROS or inflammatory markers. ${ }^{53,54}$ A recent in vitro model has shown that anthocyanin-rich supplements significantly reduced certain types of ROS, 2,2-diphenyl-1-picrylhydrazy and superoxide radicals. ${ }^{55}$ Anthocyaninrich blueberry extract induces a cellular upregulation of glutathione. Increases in glutathione mediate homeostatic levels of extracellular signal-regulated protein kinases that promote favorable learning and memory pathways. Further, anthocyanin-rich supplements can inhibit tau hyperphosphorylation in the hippocampus and improve performance on cognitive behavioral tests as shown in mouse models of AD. ${ }^{56}$

Our recent published studies ${ }^{30,32}$ demonstrated that 12-week consumption of tart cherry juice lowered systolic BP by $4 \mathrm{mmHg}$ and CRP by $25 \%$ as well as increased the plasma levels of DNA repair activity of OGG1. This supports the notion that the improvements in cognitive performance by tart cherry juice consumption may be due to its anti-inflammatory and anti-oxidative properties, and through a lowering of BP. Specifically, in vitro studies have shown that systemic inflammation impairs vascular function by interference with endothelial cell regulation, modification of vascular smooth muscle and monocyte/macrophage capacity, alteration in matrix biology, and stimulation of coagulation. This vessel damage can cause elevated BP that can eventually lead to impaired cerebral blood flow. ${ }^{57}$ In agreement with this, Kent et al. ${ }^{40}$ found that cherry juice not only significantly improved cognitive 
function but also decreased systolic BP in older adults with mild-to-moderate dementia. Relatedly, Keane et al., ${ }^{42}$ demonstrated that $60 \mathrm{ml}$ Montmorency tart cherry juice concentrate acutely lowered systolic BP over a period of three hours and modulated cerebral blood flow in the prefrontal cortex of younger men with early hypertension compared to control group.

Limitations of this study include small sample size, short duration of the tart cherry supplementation, and difference in physical activity, intake of dietary cholesterol, and MoCA at baseline between groups. Participants were asked to maintain their habitual diet and physical activity levels. However, the physical activity level significantly decreased and dietary cholesterol significantly increased in the tart cherry group and control group, respectively, indicating poor compliance with the instruction. It is important to recognize that physical activity and dietary intake vary between seasons. In order to eliminate the effects of confounding factors including physical activity, dietary cholesterol, and MoCA, the ANCOVA test was used. The majority of the participants were White, married and earned $\geq \$ 75000$ per year. Half of the participants in the tart cherry group were working while the majority of participants in the control were retired. The lack of diversity among research participants limits the generalizability of findings. In addition, we do not know the anthocyanin content of the tart cherry concentrate used for this study. Consequently, we were unable to estimate the anthocyanin intakes of the participants.

\section{Conclusion}

Cognitive function is a key determinant of independence and quality of life among older adults. Our study demonstrated that daily intake of Montmorency tart cherry juice may help improve subjective memory and cognitive abilities in older adults as evidenced by increased contentment with memory, improved visual sustained attention and spatial working memory, and reduced movement time and total errors made on new learning tasks in older adults. The present findings warrant further investigation with a larger sample size and a longer-term trial. Future studies must address the mechanisms of action, and bioavailability resulting from tart cherry exposure.

\section{Author contributions}

The authors' responsibilities were as follows: S. C. C. designed the study and conducted the research, interpreted the data, wrote the manuscript, and had primary responsibility for the final content of the manuscript; J. J. wrote the manuscript; K. D. conducted the research and edited the manuscript; R. S. W. edited the manuscript; Z. Z., a biostatistician performed the statistical analysis and interpreted the data; and all authors reviewed and approved the final manuscript.

\section{Conflicts of interest}

The authors declare that there are no conflicts of interest.

\section{Acknowledgements}

The present study was supported by the Cherry Research Committee of the Cherry Marketing Institute, a non-profit organization. Tart cherry concentrates were provided by the Cherry Marketing Institute. Funders had no role in the study design, data collection, data analysis or interpretation, or writing of the manuscript.

\section{References}

1 L. E. Hebert, J. Weuve, P. A. Scherr and D. A. Evans, Alzheimer disease in the United States (2010-2050) estimated using the 2010 census, Neurology, 2013, 80, 17781783.

2 U. S. Census Bureau, Older people projected to outnumber children for first time in U.S. history, https://www.census. gov/newsroom/press-releases/2018/cb18-41-population-projections.html, (accessed 4/26/2019, 2018).

3 D. E. Barnes and K. Yaffe, The projected effect of risk factor reduction on Alzheimer's disease prevalence, Lancet Neurol., 2011, 10, 819-828.

4 E. C. Leritz, R. E. McGlinchey, I. Kellison, J. L. Rudolph and W. P. Milberg, Cardiovascular Disease Risk Factors and Cognition in the Elderly, Curr. Cardiovasc. Risk Rep., 2011, 5, 407-412.

5 L. Kilander, H. Nyman, M. Boberg, L. Hansson and $\mathrm{H}$. Lithell, Hypertension is related to cognitive impairment: a 20-year follow-up of 999 men, Hypertension, 1998, 31, 780-786.

6 L. Kilander, H. Nyman, M. Boberg and H. Lithell, The association between low diastolic blood pressure in middle age and cognitive function in old age. A population-based study, Age Ageing, 2000, 29, 243-248.

7 L. J. Launer, K. Masaki, H. Petrovitch, D. Foley and R. J. Havlik, The association between midlife blood pressure levels and late-life cognitive function. The Honolulu-Asia Aging Study, J. Am. Med. Assoc., 1995, 274, 1846-1851.

8 N. Cherbuin, C. Reglade-Meslin, R. Kumar, P. Jacomb, S. Easteal, H. Christensen, P. Sachdev and K. J. Anstey, Risk factors of transition from normal cognition to mild cognitive disorder: the PATH through Life Study, Dementia Geriatr. Cognit. Disord., 2009, 28, 47-55.

9 D. Kritz-Silverstein, G. A. Laughlin, L. K. McEvoy and E. Barrett-Connor, Sex and Age Differences in the Association of Blood Pressure and Hypertension with Cognitive Function in the Elderly: The Rancho Bernardo Study, J. Prev. Alzheimers Dis., 2017, 4, 165-173. 
10 X. Gan, L. Wu, S. Huang, C. Zhong, H. Shi, G. Li, H. Yu, R. Howard Swerdlow, J. Xi Chen and S. S. Yan, Oxidative stress-mediated activation of extracellular signal-regulated kinase contributes to mild cognitive impairment-related mitochondrial dysfunction, Free Radicals Biol. Med., 2014, 75, 230-240.

11 M. C. McCord and E. Aizenman, The role of intracellular zinc release in aging, oxidative stress, and Alzheimer's disease, Front. Aging Neurosci., 2014, 6, 77.

12 B. D'Autreaux and M. B. Toledano, ROS as signalling molecules: mechanisms that generate specificity in ROS homeostasis, Nat. Rev. Mol. Cell Biol., 2007, 8, 813-824.

13 D. G. Loughrey, S. Lavecchia, S. Brennan, B. A. Lawlor and M. E. Kelly, The Impact of the Mediterranean Diet on the Cognitive Functioning of Healthy Older Adults: A Systematic Review and Meta-Analysis, Adv. Nutr., 2017, 8, 571-586.

14 R. J. Hardman, G. Kennedy, H. Macpherson, A. B. Scholey and A. Pipingas, Adherence to a Mediterranean-Style Diet and Effects on Cognition in Adults: A Qualitative Evaluation and Systematic Review of Longitudinal and Prospective Trials, Front. Nutr., 2016, 3, 22.

15 A. A. M. Berendsen, J. H. Kang, O. van de Rest, E. J. M. Feskens, L. de Groot and F. Grodstein, The Dietary Approaches to Stop Hypertension Diet, Cognitive Function, and Cognitive Decline in American Older Women, J. Am. Med. Dir. Assoc., 2017, 18, 427-432.

16 A. Dreiseitel, G. Korte, P. Schreier, A. Oehme, S. Locher, M. Domani, G. Hajak and P. G. Sand, Berry anthocyanins and their aglycons inhibit monoamine oxidases A and B, Pharmacol. Res., 2009, 59, 306-311.

17 J. A. Joseph, B. Shukitt-Hale, N. A. Denisova, R. L. Prior, G. Cao, A. Martin, G. Taglialatela and P. C. Bickford, Longterm dietary strawberry, spinach, or vitamin E supplementation retards the onset of age-related neuronal signaltransduction and cognitive behavioral deficits, J. Neurosci., 1998, 18, 8047-8055.

18 R. Krikorian, E. L. Boespflug, D. E. Fleck, A. L. Stein, J. D. Wightman, M. D. Shidler and S. Sadat-Hossieny, Concord grape juice supplementation and neurocognitive function in human aging, J. Agric. Food Chem., 2012, 60, 5736-5742.

19 R. Krikorian, M. D. Shidler, T. A. Nash, W. Kalt, M. R. Vinqvist-Tymchuk, B. Shukitt-Hale and J. A. Joseph, Blueberry supplementation improves memory in older adults, J. Agric. Food Chem., 2010, 58, 3996-4000.

20 B. L. Halvorsen, M. H. Carlsen, K. M. Phillips, S. K. Bohn, K. Holte, D. R. Jacobs Jr. and R. Blomhoff, Content of redoxactive compounds (ie, antioxidants) in foods consumed in the United States, Am. J. Clin. Nutr., 2006, 84, 95-135.

21 G. Desideri, C. Kwik-Uribe, D. Grassi, S. Necozione, L. Ghiadoni, D. Mastroiacovo, A. Raffaele, L. Ferri, R. Bocale, M. C. Lechiara, C. Marini and C. Ferri, Benefits in cognitive function, blood pressure, and insulin resistance through cocoa flavanol consumption in elderly subjects with mild cognitive impairment: the Cocoa,
Cognition, and Aging (CoCoA) study, Hypertension, 2012, 60, 794-801.

22 D. Mastroiacovo, C. Kwik-Uribe, D. Grassi, S. Necozione, A. Raffaele, L. Pistacchio, R. Righetti, R. Bocale, M. C. Lechiara, C. Marini, C. Ferri and G. Desideri, Cocoa flavanol consumption improves cognitive function, blood pressure control, and metabolic profile in elderly subjects: the Cocoa, Cognition, and Aging (CoCoA) Study-a randomized controlled trial, Am. J. Clin. Nutr., 2015, 101, 538-548.

23 A. M. Brickman, U. A. Khan, F. A. Provenzano, L. K. Yeung, W. Suzuki, H. Schroeter, M. Wall, R. P. Sloan and S. A. Small, Enhancing dentate gyrus function with dietary flavanols improves cognition in older adults, Nat. Neurosci., 2014, 17, 1798-1803.

24 S. T. Francis, K. Head, P. G. Morris and I. A. Macdonald, The effect of flavanol-rich cocoa on the fMRI response to a cognitive task in healthy young people, J. Cardiovasc. Pharmacol., 2006, 47(Suppl 2), S215-S220.

25 N. D. Fisher, F. A. Sorond and N. K. Hollenberg, Cocoa flavanols and brain perfusion, J. Cardiovasc. Pharmacol., 2006, 47(Suppl 2), S210-S214.

26 A. Saric, S. Sobocanec, T. Balog, B. Kusic, V. Sverko, V. Dragovic-Uzelac, B. Levaj, Z. Cosic, Z. Macak Safranko and T. Marotti, Improved antioxidant and anti-inflammatory potential in mice consuming sour cherry juice (Prunus Cerasus cv. Maraska), Plant Foods Hum. Nutr., 2009, 64, 231-237.

27 T. Traustadottir, S. S. Davies, A. A. Stock, Y. Su, C. B. Heward, L. J. Roberts 2nd and S. M. Harman, Tart cherry juice decreases oxidative stress in healthy older men and women, J. Nutr., 2009, 139, 1896-1900.

28 V. Mulabagal, G. A. Lang, D. L. DeWitt, S. S. Dalavoy and M. G. Nair, Anthocyanin content, lipid peroxidation and cyclooxygenase enzyme inhibitory activities of sweet and sour cherries, J. Agric. Food Chem., 2009, 57, 1239-1246.

29 B. Ou, K. N. Bosak, P. R. Brickner, D. G. Iezzoni and E. M. Seymour, Processed tart cherry products-comparative phytochemical content, in vitro antioxidant capacity and in vitro anti-inflammatory activity, J. Food Sci., 2012, 77, H105-H112.

30 S. C. Chai, K. Davis, Z. Zhang, L. Zha and K. F. Kirschner, Effects of Tart Cherry Juice on Biomarkers of Inflammation and Oxidative Stress in Older Adults, Nutrients, 2019, 11(2), 228.

31 S. Burkhardt, D. X. Tan, L. C. Manchester, R. Hardeland and R. J. Reiter, Detection and quantification of the antioxidant melatonin in Montmorency and Balaton tart cherries (Prunus cerasus), J. Agric. Food Chem., 2001, 49, 4898-4902.

32 S. C. Chai, K. Davis, R. S. Wright, M. F. Kuczmarski and Z. Zhang, Impact of tart cherry juice on systolic blood pressure and low-density lipoprotein cholesterol in older adults: a randomized controlled trial, Food Funct., 2018, 9, 3185-3194.

33 W. R. Pigeon, M. Carr, C. Gorman and M. L. Perlis, Effects of a tart cherry juice beverage on the sleep of older adults with insomnia: a pilot study, J. Med. Food, 2010, 13, 579-583. 
34 G. Howatson, P. G. Bell, J. Tallent, B. Middleton, M. P. McHugh and J. Ellis, Effect of tart cherry juice (Prunus cerasus) on melatonin levels and enhanced sleep quality, Eur. J. Nutr., 2012, 51, 909-916.

35 H. He, W. Dong and F. Huang, Anti-amyloidogenic and anti-apoptotic role of melatonin in Alzheimer disease, Curr. Neuropharmacol., 2010, 8, 211-217.

36 N. Thangthaeng, S. M. Poulose, S. M. Gomes, M. G. Miller, D. F. Bielinski and B. Shukitt-Hale, Tart cherry supplementation improves working memory, hippocampal inflammation, and autophagy in aged rats, Age, 2016, 38, 393404.

37 J. J. Matchynski, S. A. Lowrance, C. Pappas, J. Rossignol, N. Puckett, M. Sandstrom and G. L. Dunbar, Combinatorial treatment of tart cherry extract and essential fatty acids reduces cognitive impairments and inflammation in the mu-p75 saporin-induced mouse model of Alzheimer's disease, J. Med. Food, 2013, 16, 288-295.

38 Z. S. Nasreddine, N. A. Phillips, V. Bedirian, S. Charbonneau, V. Whitehead, I. Collin, J. L. Cummings and H. Chertkow, The Montreal Cognitive Assessment, MoCA: a brief screening tool for mild cognitive impairment, J. Am. Geriatr. Soc., 2005, 53, 695-699.

39 F. Faul, E. Erdfelder, A. Buchner and A. G. Lang, Statistical power analyses using $G^{*}$ Power 3.1: tests for correlation and regression analyses, Behav. Res. Methods, 2009, 41, 11491160.

40 K. Kent, K. Charlton, S. Roodenrys, M. Batterham, J. Potter, V. Traynor, H. Gilbert, O. Morgan and R. Richards, Consumption of anthocyanin-rich cherry juice for 12 weeks improves memory and cognition in older adults with mildto-moderate dementia, Eur. J. Nutr., 2017, 56, 333-341.

41 K. Caldwell, K. E. Charlton, S. Roodenrys and A. Jenner, Anthocyanin-rich cherry juice does not improve acute cognitive performance on RAVLT, Nutr. Neurosci., 2016, 19, 423-424.

42 K. M. Keane, C. F. Haskell-Ramsay, R. C. Veasey and G. Howatson, Montmorency Tart cherries (Prunus cerasus L.) modulate vascular function acutely, in the absence of improvement in cognitive performance, Br. J. Nutr., 2016, 116, 1935-1944.

43 L. M. Willis, B. Shukitt-Hale and J. A. Joseph, Recent advances in berry supplementation and age-related cognitive decline, Curr. Opin. Clin. Nutr. Metab. Care, 2009, 12, 91-94.

44 A. Kirakosyan, E. M. Seymour, J. Wolforth, R. McNish, P. B. Kaufman and S. F. Bolling, Tissue bioavailability of anthocyanins from whole tart cherry in healthy rats, Food Chem., 2015, 171, 26-31.

45 R. Hardeland and S. R. Pandi-Perumal, Melatonin, a potent agent in antioxidative defense: actions as a natural food constituent, gastrointestinal factor, drug and prodrug, Nutr. Metab., 2005, 2, 22.

46 R. Krikorian, T. A. Nash, M. D. Shidler, B. Shukitt-Hale and J. A. Joseph, Concord grape juice supplementation improves memory function in older adults with mild cognitive impairment, Br. J. Nutr., 2010, 103, 730-734.

47 G. Casadesus, B. Shukitt-Hale, H. M. Stellwagen, X. Zhu, H. G. Lee, M. A. Smith and J. A. Joseph, Modulation of hippocampal plasticity and cognitive behavior by short-term blueberry supplementation in aged rats, Nutr. Neurosci., 2004, 7, 309-316.

48 C. Andres-Lacueva, B. Shukitt-Hale, R. L. Galli, O. Jauregui, R. M. Lamuela-Raventos and J. A. Joseph, Anthocyanins in aged blueberry-fed rats are found centrally and may enhance memory, Nutr. Neurosci., 2005, 8, 111-120.

49 J. L. Bowtell, Z. Aboo-Bakkar, M. E. Conway, A. R. Adlam and J. Fulford, Enhanced task-related brain activation and resting perfusion in healthy older adults after chronic blueberry supplementation, Appl. Physiol., Nutr., Metab., 2017, 42, 773-779.

50 M. Chalopin, A. Tesse, M. C. Martinez, D. Rognan, J. F. Arnal and R. Andriantsitohaina, Estrogen receptor alpha as a key target of red wine polyphenols action on the endothelium, PLoS One, 2010, 5, e8554.

51 J. Lee, N. Torosyan and D. H. Silverman, Examining the impact of grape consumption on brain metabolism and cognitive function in patients with mild decline in cognition: A double-blinded placebo controlled pilot study, Exp. Gerontol., 2017, 87, 121-128.

52 P. Davalli, T. Mitic, A. Caporali, A. Lauriola and D. D'Arca, ROS, Cell Senescence, and Novel Molecular Mechanisms in Aging and Age-Related Diseases, Oxid. Med. Cell. Longevity, 2016, 2016, 3565127.

53 J. P. Spencer, Flavonoids and brain health: multiple effects underpinned by common mechanisms, Genes Nutr., 2009, 4, 243-250.

54 S. Subash, M. M. Essa, S. Al-Adawi, M. A. Memon, T. Manivasagam and M. Akbar, Neuroprotective effects of berry fruits on neurodegenerative diseases, Neural Regener. Res., 2014, 9, 1557-1566.

55 G. Casedas, F. Les, M. P. Gomez-Serranillos, C. Smith and V. Lopez, Bioactive and functional properties of sour cherry juice (Prunus cerasus), Food Funct., 2016, 7, 4675-4682.

56 T. Ali, M. J. Kim, S. U. Rehman, A. Ahmad and M. O. Kim, Anthocyanin-Loaded PEG-Gold Nanoparticles Enhanced the Neuroprotection of Anthocyanins in an Abeta1-42 Mouse Model of Alzheimer's Disease, Mol. Neurobiol., 2017, 54, 6490-6506.

57 C. Iadecola and R. F. Gottesman, Neurovascular and Cognitive Dysfunction in Hypertension, Circ. Res., 2019, 124, 1025-1044. 